Acta Physica Academiae Scientiarum Hungaricae, Tomus 24 (4), pp. 325-330 (1968)

\title{
USE OF DELTA FUNCTIONS IN GENERAL RELATIVITY FOR DETERMINATION OF THE INTEGRATION CONSTANTS
}

\author{
By \\ E. Schmutzer \\ INSTITUTE FOR THEORETICAL PHYSICS OF THE UNIVERSITY OF JENA, JENA, DDR
}

(Presented by A. Kónya. - Received 4. IV. 1967)

\begin{abstract}
During the integration of the Einstein-Maxwell equations, integration constants appear and their interpretation is often very difficult. There is therefore, a requirement for a calculus of delta functions which will automatically relate integration constants to sources. In this paper a calculus of this kind is developed and applied to the spherically symmetric problem. In this way we get a method of distinction between pure mathematical and physical singularities.
\end{abstract}

\section{Calculus of delta functions}

In this paper a calculus of delta functions suitable for certain physical problems is presented. The work here extends the use of delta functions to be found in a paper by Rosen and Shamir [1] and a book by INFELD and Plebansky [2].

A general delta tensor of the rank $k$ in space-time is defined by

$$
\delta^{n_{1}} \ldots n_{k}\left(x^{j}, \xi^{j}\right)=\delta^{n_{1} \ldots n_{k}}\left(\xi^{j}, x^{j}\right)=\left\{\begin{array}{c}
0 \text { for } x^{j} \neq \xi^{j} \\
\infty \text { for } x^{j}=\xi^{j}
\end{array}\right.
$$

( $k$ is an integer between 1 and 4). The strength of the singularity is of such a kind that

$$
i \int_{V_{a-k}} \delta^{n_{1} \ldots n_{k}}\left(x^{j}, \xi^{j}\right) d f_{n_{1}} \ldots n_{k}\left(x^{j}\right)=1
$$

is valid if the singularity is situated in the $(4-k)$-dimensional subspace over which the integral is taken. $d f_{n_{1}} \ldots n_{k}$ is the surface-element pseudo-tensor of rank $k$. A consequence of (1) and (2) is the equation

$$
i \int_{V_{t-k}} F\left(x^{j}\right) \delta^{n_{1}} \ldots n_{k}\left(x^{j}, \xi^{j}\right) d f_{n_{1}} \ldots n_{k}\left(x^{j}\right)=F\left(\xi^{j}\right) .
$$

(The factor $i$ in the last two equations is a consequence of definition of $d f_{n_{1}} \ldots n_{k}$ ) Two cases are particularly interesting: namely if the delta tensor is of the rank $0(k=0)$ we get

$$
\int_{V_{1}} F\left(x^{j}\right) \delta\left(x^{j}, \xi^{j}\right) d^{(4)} f\left(x^{j}\right)=F\left(\xi^{j}\right)
$$


and if the delta tensor is of the rank $1(k=1)$, we get

$$
i \int_{V_{3}} F\left(x^{j}\right) \delta^{k}\left(x^{j}, \xi^{j}\right) d f_{k}\left(x^{j}\right)=F\left(\xi^{j}\right)
$$

In the first case the delta tensor is called Dirac's delta function. In the second case it is called a surface delta function. Our further investigations refer to Dirac's delta function.

First we study the 1-dimensional case. Heaviside's step function is defined by

$$
\Theta(x)=\left\{\begin{array}{rr}
-\frac{1}{2} & \text { for } x<0 \\
0 & \text { for } x=0 \\
\frac{1}{2} & \text { for } x>0
\end{array}\right.
$$

Bo that the relations

a) $\Theta(x)^{\prime}=\delta(x)$, b) $\left.\Theta(x)+\Theta(-x)=0, c\right) \Theta(x) \Theta(-x)=\left\{\begin{array}{r}-\frac{1}{4} \text { for } x<0 \\ 0 \text { for } x=0(7) \\ -\frac{1}{4} \text { for } x>0\end{array}\right.$

hold. Dirac's delta function satisfies the differential equation

$$
x \delta^{\prime}(x)+\delta(x)=0 .
$$

The 3-dimensional delta function is given by

$$
\delta(\vec{r})=\delta(x) \delta(y) \delta(z) \quad\left(r=\sqrt{x^{2}+y^{2}+z^{2}}\right) .
$$

This quantity allows the definition of the 3-dimensional radial delta function for which the equations

$$
\delta(r)=4 \pi r^{2} \delta(\vec{r}),
$$

$$
\text { a) } \delta(r)=\left\{\begin{array}{r}
\infty \text { for } r=0 \\
0 \text { for } r>0
\end{array} \quad, \quad \text { b) } \int_{0}^{\infty} \delta(r) d r=1\right.
$$

are valid. The relation

$$
\delta(r)=2 \bar{\Theta}(r)^{\prime}
$$

exists between the delta function and the radial step function

$$
\bar{\Theta}(r)=\left\{\begin{array}{c}
0 \text { for } r=0 \\
\frac{1}{2} \text { for } r>0 .
\end{array}\right.
$$


By differentiation one verifies the important equation

$$
\Delta\left(\frac{\bar{\Theta}(r)}{2 \pi r}\right)=-\delta(\vec{r})
$$

where $\Delta$ is the Laplace operator in spherical polar coordinates. In distribution theory literature, this is replaced by the notation

$$
\Delta\left(\frac{1}{4 \pi r}\right)=-\delta(\vec{r})
$$

The 2-dimensional radial delta function is defined by

$$
\delta(\sigma)=2 \pi \sigma \delta(x) \delta(y) \quad\left(\sigma=\sqrt{x^{2}+y^{2}}\right) .
$$

For this the relations

$$
\text { a) } \delta(\sigma)=\left\{\begin{array}{r}
\infty \text { for } \sigma=0 \\
0 \text { for } \sigma>0
\end{array}, \quad \text { b) } \int_{0}^{\infty} \delta(\sigma) d \sigma=1\right.
$$

are valid. By calculation one verifies that

$$
\left(\frac{\partial^{2}}{\partial x^{2}}+\frac{\partial^{2}}{\partial y^{2}}\right)\left(-\frac{\bar{\Theta}(\sigma) \ln \sigma}{2 \pi}\right)=-\delta(x) \delta(y) .
$$

\section{Investigation of Reissner-Weyl - Schwarzschild field}

The square of the line element is used in the form

$$
\begin{array}{r}
(d s)^{2}=e^{\mu(r)}(d r)^{2}+r^{2}\left[\sin ^{2} \Theta(d \varphi)^{2}+(d \Theta)^{2}\right]-e^{v(r)}\left(d x^{4}\right)^{2} \\
\left(x^{1}=r, x^{2}=\varphi, x^{3}=\Theta\right)
\end{array}
$$

We write the field equations in the form

a) $R_{t}^{j}=\varkappa\left(T_{i}^{j}-\frac{1}{2} g_{i}^{j} T_{m}^{m}\right), \quad$ (Einstein equation)

b) $\left(E^{\mu} \sqrt{\overline{-\mathbf{g}}}\right)_{1 \mu}=\varrho \sqrt{\overline{-\mathbf{g}}}$

c) $\left(E_{3} \sqrt{-g_{44}}\right)_{, 1}=\left(E_{1} \sqrt{-g_{44}}\right)_{, 3}=0, \quad\left(E_{2} \sqrt{-g_{44}}\right)_{, 1}=\left(E_{1} \sqrt{-g_{44}}\right)_{, 2}, \quad$ (Maxwell

$$
\left.\left(E_{3} \sqrt{-g_{44}}\right)_{, 2}=\left(E_{2} \sqrt{-g_{44}}\right)_{, 3}, \quad\left(\stackrel{4}{g}=-\left|g_{i j}\right|, \stackrel{3}{g}=-\left|g_{\mu v}\right|\right) \quad \text { equation }\right)
$$

taking into account that we have to treat a static problem. (Greek indices run from 1 to 3 , latin indices from 1 to 4.) $E^{\mu}=g^{\mu \nu} E_{y}$ is the 3-dimensional electric field strength, and $\varrho$ is the 3 -dimensional charge density. 
The Einstein equations can be written in the following way:

$$
\begin{aligned}
& \text { a) } \frac{\nu^{\prime \prime}}{2}+\frac{\nu^{\prime 2}}{4}-\frac{\mu^{\prime}}{r}-\frac{\nu^{\prime} \mu^{\prime}}{4}=\varkappa e^{\mu}\left(T_{1}^{1}-\frac{1}{2} T_{m}^{m}\right), \\
& \text { b) } \frac{\nu^{\prime}-\mu^{\prime}}{2} r+1-e^{\mu}=\varkappa r^{2} e^{\mu}\left(T_{2}^{2}-\frac{1}{2} T_{m}^{m}\right) \\
& \text { c) } \frac{\nu^{\prime}-\mu^{\prime}}{2} r+1-e^{\mu}=\varkappa r^{2} e^{\mu}\left(T_{3}^{3}-\frac{1}{2} T_{m}^{m}\right) \\
& \text { d) } \frac{\nu^{\prime \prime}}{2}+\frac{\nu^{\prime 2}}{4}+\frac{\nu^{\prime}}{r}-\frac{\nu^{\prime} \mu^{\prime}}{4}=\varkappa e^{\mu}\left(T_{4}^{4}-\frac{1}{2} T_{m}^{m}\right) .
\end{aligned}
$$

From the second and third it follows that

$$
T_{2}^{2}=T_{3}^{3},
$$

while the first and the fourth yield

$$
\frac{\nu^{\prime}+\mu^{\prime}}{r}=x e^{\mu}\left(T_{4}^{4}-T_{1}^{1}\right) .
$$

Using the abbreviation

$$
\gamma=e^{-\mu}
$$

we get from (20) after some calculation

$$
\begin{gathered}
T_{m}^{m}=-\frac{\gamma}{\varkappa}\left[\nu^{\prime \prime}+\frac{\nu^{\prime 2}}{2}+\frac{2 \nu^{\prime}}{r}\right]+\frac{1}{\varkappa}\left[\frac{2}{r^{2}}(1-\gamma)-\frac{\nu^{\prime} \gamma^{\prime}}{2}-\frac{2 \gamma^{\prime}}{r}\right], \\
T_{4}^{4}=\frac{1}{\varkappa}\left[-\frac{\gamma^{\prime}}{r}+\frac{1}{r^{2}}(1-\gamma)\right], \\
T_{1}^{1}=\frac{1}{\varkappa}\left[\frac{1}{r^{2}}(1-\gamma)-\frac{\nu^{\prime} \gamma}{r}\right], \\
T_{2}^{2}=-\frac{\gamma}{2 \varkappa}\left[\nu^{\prime \prime}+\frac{\nu^{\prime 2}}{2}+\frac{\nu^{\prime}}{r} \mid-\frac{\gamma^{\prime}}{2 \varkappa}\left[\frac{1}{r}+-\frac{\nu^{\prime}}{2}\right] .\right.
\end{gathered}
$$

The energy tensor is now split into an electromagnetic part $E_{i}^{j}$ and a remainder part $\Theta_{l}^{j}$ :

where

$$
T_{i}^{j}=E_{i}^{j}+\Theta_{i}^{j},
$$

$$
\left(E_{i}^{j}\right)=\left(\begin{array}{c|c}
E_{\mu} E^{v}-\frac{1}{2} g_{\mu}^{\prime} E_{\lambda} E^{\lambda} & 0 \\
\hline 0 & \frac{1}{2} E_{\lambda} E^{\lambda}
\end{array}\right), \quad\left(\Theta_{i}^{j}\right)=\left(\begin{array}{cccc}
\alpha & 0 & 0 & 0 \\
0 & \beta & 0 & 0 \\
0 & 0 & \beta & 0 \\
0 & 0 & 0 & \mu c^{2}
\end{array}\right) .
$$


An electrical point singularity with the charge $e$ must be described by

$$
\varrho(r)=\frac{e}{4 \pi r^{2} e^{\mu / 2}} \delta(r)
$$

because the equation

$$
\int \varrho d^{(3)} f=\int \varrho \sqrt{-g^{3}} d^{(3)} x=e \int_{0}^{\infty} \delta(r) d r=e
$$

must be valid.

The integration of the Maxwell equations yields

$$
E_{2}=E_{3}=0, E_{1}=\frac{e \bar{\theta}(r) e^{\mu / 2}}{2 \pi r^{2}}
$$

so that

follows.

$$
\begin{aligned}
& E_{1}^{1}=E_{4}^{4}=\frac{1}{2} E_{1} E^{1}=\frac{e^{2} \bar{\theta}(r)^{2}}{8 \pi^{2} r^{4}} \\
& E_{2}^{2}=E_{3}^{3}=-\frac{1}{2} E_{1} E^{1}=-\frac{e^{2} \bar{\theta}(r)^{2}}{8 \pi^{2} r^{4}}
\end{aligned}
$$

For integration of (25) we choose for a point singularity with the mass $M$ the rest mass density

$$
\mu=\frac{M \delta(r)}{4 \pi r^{2}}
$$

Using (33) we find

$$
(\gamma r)^{\prime}=1-\frac{\varkappa M c^{2} \delta(r)}{4 \pi}-\frac{\varkappa e^{2} \bar{\theta}(r)^{2}}{8 \pi^{2} r^{2}}
$$

and further by integration

$$
\gamma=1-\frac{\varkappa M c^{2} \bar{\theta}(r)}{2 \pi r}+\frac{\varkappa e^{2} \bar{\theta}(r)^{2}}{8 \pi^{2} r^{2}}+\frac{\varkappa e^{2}}{8 \pi^{2} r} \int_{0}^{r} \bar{\theta}(r) \delta^{\prime}(r) d r .
$$

Outside the singularity this is the well known result

$$
\gamma=1-\frac{x M c^{2}}{4 \pi r}+\frac{x e^{2}}{32 \pi^{2} r^{2}}
$$

$\mathrm{U}_{\mathrm{p}}$ to this point the stress distribution in the singularity is not fixed. Further assumptions about $v(r)$ would be necessary. The formula $v+\mu=0$, which holds outside the singularity, leads to non-physical stress inside the singularity. 


\title{
REFERENCES
}

1. N. Rosen and H. Shamir, Rev. Mod. Phys., 29, 429, 195 i.

2. L. Infeld and J. Plebanski, Motion and Relativity. Warsaw, 1960.

\section{ПРИМЕНЕНИЕ $\delta$-ФУНКЦИЙ В ТЕОРИИ ОБЩЕЙ ОТНОСИТЕЛЬНОСТИ ДЛЯ ОПРЕДЕЛЕНИЯ ПОСТОЯННЫХ ИНТЕГРИРОВАНИЯ}

\author{
Е. ШМУЦЕР \\ P е $з$ ю
}

При интегрировании уравнений Эйнштейна-- Максвелла появляются постоянные интегрирования, интерпретация которых часто представляется очень трудной. Отсюда возникает потребность применения в вычислениях $\delta$-функций, которые автоматически указывают на происхождение этих постоянных. В данной работе развивается метод такого характера, дается его применение в случае проо̆лемы, обладающей сферической симметрией. Таким путем нами дается метод для различия между чисто математической и физической сингулярностями. 